\title{
Original
}

\section{Organización de la atención médica en la Epidemia de Dengue hemorrágico en el Hospital "Dr. Enrique Baltodano" de Liberia, 2003}

Anabelle Alfaro Obando, Mónica Guardia Caldera, Cristian Angulo Jaubert

\section{Resumen}

Justificación y objetivos: El dengue es una enfermedad endémica, lo que implica que todos los años se presentan brotes en el país que justifican establecer una organización especial de los servicios de salud para garantizar una atención oportuna a la población.

Método: Se realizó un estudio transversal descriptivo en 1153 casos internados en el Hospital “Dr. Enrique Baltodano” de la ciudad de Liberia, durante 2003. Se efectuó un análisis descriptivo de sexo, edad, estancia hospitalaria y diagnóstico de egreso. Se describió la organización de la atención y el manejo médico de los pacientes en una epidemia de dengue. En ese año el laboratorio de referencia de dengue de Instituto Costarricense de Investigación en Nutrición y Salud (INCIENSA) detectó la circulación del virus dengue serotipo 2. No se reportaron defunciones en el país por dengue hemorrágico.

Resultados: Se estudiaron 1153 pacientes hospitalizados por dengue, 549 (47.6\%) del sexo masculino y 604 (52.4\%) del sexo femenino. La edad promedio fue de 33 años, con un rango de 2 a 94, de los cuales 52 (5\%) cumplieron criterios para dengue hemorrágico; del resto, 949 (82\%) tenían conteo de plaquetas menores de 100.000/. $\mathrm{mm}^{3}$, y 152 (13\%) presentaron manifestaciones hemorrágicas; no se detectó en estos dos grupos datos de extravasación de plasma por ultrasonido. No se presentaron complicaciones ni fallecidos durante la hospitalización. La estancia hospitalaria promedio fue de 2.26 días (IC95\%= 2.32 - 2.19).

Conclusión: Los resultados obtenidos evidencian la necesidad de contar con una estrategia para organizar la atención en epidemias de dengue. Esto garantiza una tasa de letalidad del $0 \%$ y estancias hospitalarias bajas. Lo anterior se logra mediante la identificación temprana de

Unidad de Atención de Dengue, Hospital Dr. Enrique Baltodano.

Abreviaturas: $\quad$ IC95\%; Intervalo de confianza al $95 \%$; OMS, Organización Mundial de la Salud; OPS, Organización Panamericana de la Salud

Correspondencia: Dra. Anabelle Alfaro Obando e-mail:

anabellealfaro@gmail.com

ISSN 0001-6002/2006/48/4/185-189 Acta Médica Costarricense, (C2006 Colegio de Médicos y Cirujanos criterios de ingreso, utilizando datos clínicos y de laboratorio. Esta metodología disminuye los costos económicos y está al alcance de todos los servicios de salud.

Descriptores: Dengue clásico con trombocitopenia, tratamiento del dengue hemorrágico, ultrasonido en dengue.

Key words: Clasic dengue fever, treatment of dengue, hemorragic dengue, ultrasonid in dengue.

Recibido: 29 de marzo de 2006

Aceptado: 8 de agosto de 2006

Actualmente, el dengue es la enfermedad viral trasmitida a los humanos por mosquitos, que representa uno de los mayores problemas de salud pública en los países tropicales y subtropicales, con predominio en las áreas urbanas y periurbanas ${ }^{1}$. 
En Costa Rica, desde 1993 el dengue es una enfermedad endémica en las costas del Pacífico y del Caribe. Durante estos años y hasta julio de 2005 se han notificado 387 pacientes con dengue hemorrágico. Antes de 1999 se reportaron cinco pacientes que fallecieron con dengue hemorrágico ${ }^{2}$.

En 2003 se notificaron en el país 19.701 casos de dengue, de los cuales 9.309 pertenecían a la región Chorotega. En esta región la epidemia de dengue se inició en las áreas de salud de Carrillo, Nicoya y Santa Cruz y se extendió en agosto a Liberia, Cañas, Bagaces, Tilarán y La Cruz; estas áreas pertenecen al núcleo de atención de Liberia. La región está cubierta por 10 áreas de salud y tiene tres hospitales, para una población de 348.263 habitantes ${ }^{3}$, según datos del Centro de Estadística y Censos (2003).

Durante esta epidemia, el laboratorio de referencia para dengue del Instituto Costarricense de Investigación en Nutrición y Salud, detectó la circulación del serotipo 2 del virus del dengue.

La gran dificultad para el control del Aedes aegyti en dichas localidades, la presentación histórica de casos de dengue clásico, la alta densidad de población y las urbanizaciones no planificadas, constituyeron el factor desencadenante en la aparición de la epidemia y su gravedad clínica ${ }^{3}$.

La constante y masiva presentación de casos hace del dengue una de las pocas enfermedades que justifican establecer una organización especial de los servicios de salud. Es preciso garantizar un tratamiento oportuno a la población con un brote de dengue, con seguimiento médico diario y hospitalización de quienes presentan algunos de los criterios que ameritan el ingreso a las unidades de atención ${ }^{4}$.

\section{Material y métodos}

El presente estudio es transversal descriptivo; toma en cuenta Consulta Externa y la Unidad de Dengue del Hospital de Liberia, en el Pacífico Seco de Costa Rica:

1. Consulta Externa: servicio definido para la consulta de casos sospechosos de dengue. En ella se atendió a todos los pacientes que cumplieron con la definición de caso sospechoso de dengue establecido en la norma ${ }^{5}$. Los pacientes fueron atendidos diariamente por un médico general, hasta cumplir 72 horas después del descenso súbito y definitivo de la fiebre.

Los pacientes con al menos uno de los siguientes criterios de ingreso fueron referidos al Hospital Dr. Enrique Baltodano de Liberia: plaquetas menores de $100.000 / \mathrm{mm}^{3}$, evidencia de sangrado o ataque al estado general y edemas en niños.
2. Unidad de atención de los casos con dengue: servicio definido para la hospitalización de los pacientes con criterios de ingreso provenientes de la consulta de control de los casos de dengue del primer nivel de atención. La Unidad de dengue se integró con enfermeras profesionales y auxiliares de enfermería, y un médico general con experiencia en el manejo de los casos.

El número de camas asignadas correspondió al 10\% de la población afectada por el brote de dengue, con un espacio libre para aumentar el cupo, de ser necesario. Se habilitó el espacio físico con medidas de protección para evitar la entrada de mosquitos (ventanales y puertas protegidas con cedazo o malla fina).

El equipo médico utilizado incluyó: estetoscopios; esfigmomanómetros con brazaletes para neonatos, niños o adultos, y equipo portátil de diagnóstico.

Todo paciente hospitalizado se mantuvo con una vía intravenosa permeable para ser utilizada si así se requería. Se indicó como analgésico antipirético, paracetamol en dosis usual. La alimentación e hidratación oral se administró a libre demanda.

Los pacientes fueron clasificados con una plaquita numerada de color rojo o verde, de acuerdo con el día de evolución de la fiebre. Para los días 1, 2, 6 y 7 se empleó una placa verde, y para los días 3, 4, 5, una roja. Este tiempo corresponde al período entre las 24 y las 72 horas después de la caída súbita y definitiva de la fiebre.

A los pacientes hospitalizados se les practicó diariamente un hemograma.

A los pacientes con determinación de plaquetas menor de $100.000 / \mathrm{mm}^{3}$ se les hizo un estudio ultrasonográfico de abdomen y tórax para detectar signos de extravasación de plasma. El estudio se efectuó 24 horas después de la caída definitiva de la fiebre8, lo que permitió clasificar a los pacientes hospitalizados en dos grupos: los que extravasan plasma y los que a pesar de tener plaquetas por debajo de $100.000 / \mathrm{mm}^{3}$, no lo hacen.

Los pacientes clasificados con dengue hemorrágico por ultrasonido requirieron vigilancia de cerca y toma de signos vitales cada 30 minutos, entre las 24 y las 72 horas después de presentado el descenso súbito de la fiebre, con el fin de detectar la presencia de signos tempranos de hipovolemia.

Las soluciones parenterales utilizadas fueron: en adultos, solución Dacca, y en niños, solución polielectrolítica, conocida como solución 90 en Costa Rica y solución Pizarro en otros países. La solución Dacca, usada en adultos, está constituida por (g/L): $\mathrm{NaCl} 5, \mathrm{KCl} 1$, acetato de $\mathrm{Na}$ 6.53, dextrosa 10, lo que proporciona (mmol/L): Na 133, K13, Cl 98, HCO3 48 y dextrosa 56 ${ }^{6}$. La solución polielectrolítica para niños está constituida por (g/L): $\mathrm{NaCl} 3.5, \mathrm{KCl}$ 1.5, acetato de $\mathrm{Na} 4.8$ y dextrosa 20, lo que proporciona (mmol/L): Na 90, K 20, Cl 80, HCO3 30 y dextrosa 111. 
Se reservó concentrado de plaquetas para todo paciente con plaquetas menores de $50.000 / \mathrm{mm}^{3}$. Si se presentaba un sangrado importante que pusiera en peligro la vida, se transfundía concentrado de plaquetas, a razón de a 0.1 unidad $/ \mathrm{Kg}$.

Todos los pacientes permanecieron hospitalizados hasta cumplir con los siguientes criterios de egreso: conteo de plaquetas mayor de $100.000 / \mathrm{mm}^{3}$, ausencia de sangrado, transcurso de 72 horas después del descenso de la fiebre.

Para formular el diagnóstico de dengue hemorrágico se siguieron los criterios recomendados por la Organización Mundial de la Salud (OMS) ${ }^{7}$.

Al recopilar la información se utilizó el expediente médico con hoja de evolución diaria y hoja de informe diario del diagnóstico y condición clínica del paciente ${ }^{4}$.

\section{Selección de pacientes}

Se seleccionaron todos los pacientes hospitalizados con diagnóstico de dengue en Liberia, en 2003. Se estudió un total de 1153 casos que cumplieron con la información requerida. Los pacientes pertenecían a ambos sexos y tuvieron edades superiores a los 2 meses.

Para el análisis de los datos, en la primera etapa se estimaron las frecuencias y proporciones de las variables por grupo de edad, y los costos de la atención.

\section{Resultados}

La Región Chorotega notificó 9.309 casos de dengue atendidos con valoración diaria en Consulta Externa. Solo 1153 (12\%) cumplieron en algún momento de la evolución con al menos uno de los criterios de ingreso. La distribución de los casos hospitalizados según el sexo correspondió a 604 (52.4\%) mujeres, y 549 (47.6\%) hombres. La edad promedio fue de 33 años, con un rango de 2 a 94 (Cuadro 1). La estancia promedio fue de 2.26 días (IC95\%= 2.32 2.19).

En el diagnóstico de dengue hemorrágico se siguieron los criterios recomendados por la $\mathrm{OMS}^{6}$. Por medio del ultrasonido se detectó derrame pleural o ascitis en todos los pacientes hospitalizados con trombocitopenia menor de $100.000 / \mathrm{mm}^{3}$. Solo 52 (5\%) cumplieron con criterios para clasificarlos como dengue hemorrágico; 48 pacientes (94\%) fueron clasificados como dengue hemorrágico grado I, uno (2\%) como grado II (dengue hemorrágico con manifestaciones hemorrágicas) y tres (6\%) como grado III (dengue hemorrágico con shock reversible).

El costo en la organización de la atención, tanto en Consulta Externa como en la atención de los hospitalizados fue de 518 millones de colones (\$1.245 192)(\$416=\$1 al tipo de cambio en 2003).

\begin{tabular}{|lcc|}
\hline $\begin{array}{c}\text { Cuadro 1. Distribución de casos de dengue } \\
\text { hospitalizados, según grupo de edad, } \\
\text { Hospital Dr. Enrique Baltodano, 2003 }\end{array}$ \\
\hline Edad & $\mathrm{N}$ & $\%$ \\
\hline Menor de 1 año & 9 & 1 \\
1 a 10 & 105 & 9 \\
11 a 20 & 242 & 22 \\
21 a 30 & 239 & 20 \\
31 a 40 & 173 & 15 \\
41 a 50 & 158 & 14 \\
51 a 60 & 97 & 8 \\
Mayor de 60 & 130 & 11 \\
\hline Total & 1153 & 100 \\
\hline Fuente: Expedientes & médicos, Hospital & Dr. Enrique \\
Baltodano, 2003 & & \\
\hline
\end{tabular}

\section{Discusión}

La edad promedio de los pacientes ingresados en Liberia fue muy similar a la observada (38 años) en la epidemia de dengue hemorrágico de Puerto Rico (1990-1991) y en Cuba (2001) ${ }^{9}$. La estancia promedio de 2.26 días fue menor a la informada en las Filipinas, de 5.6 días ${ }^{1}$.

La realización de un estudio ultrasonográfico a todos los pacientes diagnosticados como posible caso de dengue hemorrágico (trombocitopenia menor de 100.000/mm) ${ }^{3}$ permitió clasificarlos en dos grupos: 1.- los que extravasan plasma, y 2.- los que no lo hacen; esto disminuyó en forma importante las estancias hospitalarias y sus $\operatorname{costos}^{10}$.

A diferencia de lo informado en Indonesia, donde se realizaban estudios diarios a los pacientes con conteo de plaquetas de 100,000 a $150,000 / \mathrm{mm}^{3}$, hasta detectar la extravasación de plasma ${ }^{11}$, fue necesario practicar un estudio ultrasonográfico 24 horas después del descenso de la fiebre, a quienes tenían plaquetas menores de 100,000/ $\mathrm{mm}^{3}$.

La presencia de taquicardia asociada a disminución de la presión arterial media en adultos, y únicamente taquicardia en niños, son los signos tempranos de shock reversible. Esto ha sustituido la práctica de realizar determinaciones frecuentes del valor del hematocrito para detectar una elevación de más del $20 \%$ en los pacientes, con el fin de confirmar el diagnóstico de dengue hemorrágico, como lo recomienda la Organización Panamericana de la Salud $(\mathrm{OPS})^{12}$. Se considera más oportuna una valoración clínica que un resultado de laboratorio.

Durante el internamiento solo tres pacientes presentaron inestabilidad hemodinámica, lo que señala la importancia de la hospitalización temprana para evitar manejos agresivos del síndrome de shock refractario por dengue hemorrágico, con falla multiorgánica y un riesgo de mortalidad de hasta el $47 \%{ }^{13}$. 
Las soluciones para uso endovenoso utilizadas durante el shock reversible por dengue hemorrágico son las mismas empleadas en el manejo de la deshidratación grave por cólera: solución Dacca en adultos, o solución 90 en niños ${ }^{14}$. Su uso ha permitido la infusión de grandes volúmenes sin que haya congestión pulmonar, complicación presentada en pacientes con acidemia, en los que se utilizan importantes cantidades de solución salina al $0.9 \%{ }^{15}$. Conviene destacar que no existe una cantidad de soluciones parenterales exacta por administrar al paciente, durante el tiempo cuando se presenta el aumento masivo en la permeabilidad capilar sistémica con la consecuente hipovolemia, como lo describe la OPS 3 .

No se reportan investigaciones referentes al beneficio de utilizar plasma en el dengue. Existe un metaanálisis ${ }^{16} \mathrm{y}$ un estudio en los que se compara su empleo en pacientes de terapia intensiva, sin que se justificar su uso ${ }^{17}$.

En los tres pacientes con sindrome de shock por dengue hemorrágico grado III no fue necesario el uso de plasma en el manejo, tal y como lo recomienda la OPS ${ }^{3}$. Se considera que este no repone el volumen intravascular y pasa al espacio extravascular perpetuando la hipovolemia. Al desaparecer el aumento en la permeabilidad vascular, la albúmina queda atrapada en el tercer espacio, en donde al aumentar el poder oncótico se mantiene la ascitis, el hidrotórax y el edema.

La utilización de un monitoreo no invasor, como la presión arterial media de acuerdo con edad y sexo en niños ${ }^{18}$, y en adultos mayor de $70 \mathrm{mmHg}$, ha permitido un adecuado manejo de líquidos y ha evitado las complicaciones descritas cuando se utiliza la presión de pulso, las cuales ameritan el uso de diuréticos por sobrecarga de volumen ${ }^{19}$.

El 94\% de los pacientes con dengue hemorrágico no presentó inestabilidad hemodinámica y no fue necesario suministrarles soluciones parenterales. Con monitoreo de la presión arterial media y la frecuencia cardiaca y vigilancia cercana en las unidades de dengue, estos pacientes se mantuvieron estables, con abundante hidratación oral y dieta corriente, tal y como se efectúa en algunos países ${ }^{20}$.

La detección temprana llevada a cabo en la consulta externa de las localidades con brotes de dengue y la aplicación de los criterios de ingreso utilizados, permiten que el país mantenga una de las tasas de mortalidad más bajas del mundo por síndrome de shock por dengue hemorrágico.

\section{Agradecimientos}

Al personal de Salud del Hospital “Dr. Enrique Baltodano” de Liberia, por la dedicación y calidez brindada a los pacientes.
Al Dr. Daniel Pizarro Torres, por la revisión y correcciones del manuscrito.

\section{Abstract}

Aim: Dengue is an endemic disease that causes outbreaks in the country every year. This validates the requirement of a special organization of Health Service that would guarantee immediate attention to the population.

Methods: A transversal descriptive study was done in 1153 inpatients at Dr. Enrique Baltodano Hospital throughout 2003. The descriptive phase was done by sex, age, admission stay, and discharge diagnosis. The patient assessment and management in a dengue epidemic is described. The reference laboratory for dengue was INCIENSA laboratory, which detected serotype 2 dengue circulation. No deceased were reported in the country due to dengue hemorrhagic fever.

Results: The study population was 1153 dengue patients, 549 (47.6\%) males and 604 (52.4\%) females. The mean age was 33 years with a range from 2 to 94 years, from which 52 (5\%) satisfied the criteria for dengue hemorrhagic fever, 949 (82\%) had platelet count less than $100.000 / \mathrm{mm}^{3}, 152$ (13\%) presenting hemorrhagic manifestations. There were no complications throughout the hospital stay. The mean admission stay was 2.26 days (IC95\%=2.32- 2.19).

Conclusions: These results show the need of a strategy for the management of patients during dengue epidemics. This guarantees a death rate of $0 \%$ and a shorter admission stay. These results were achieved by means of early identification of the admission criterion and using the clinical and laboratory signs for dengue diagnosis. The economic impact and abolition of lethality can be reached by every Health Service

\section{Referencias}

Carlos CC, Oishi K, Cinco MT, Mapua CA, Inoue S, Cruz DJ et al. Comparison of clinical features and hematologic abnormalities between dengue fever and dengue hemorrhagic fever among children in the Philippines. Am.J Trop.Med Hyg. 2005;73: 435-40.

2. Ministerio de Salud de Costa Rica. Departamento de Vigilancia Epidemiológica.1999-2005.

3. OPS. Dengue y dengue hemorrágico en las Américas. Guías para su prevención y control. Publicación Científica 1995; 548:73-04.

4. CCSS. Guía para la organización de la atención y manejo de los pacientes con dengue clásico y dengue hemorrágico. 2002, 3-13.

5. Ministerio de Salud. Comisión Interinstitucional de Dengue. Normas técnicas para el control del dengue y dengue hemorrágico.2000: 2106.

6. Pizarro D. Tratamiento y prevención de la enfermedad diarreica aguda. Bol Med Infant Mex. 1991; 48: 699-09. 
Boletín Epidemiológico. Resurgimiento del dengue en las Américas.1997;18 2-5.

8. Rigau-Pérez JG. Clinical manifestations of dengue hemorrhagic fever in Puerto Rico, 1990-1991. Puerto Rico Association of Epidemiologists. Pan Am J Publ Health. 1997, 1 381-88.

9. González D, Castro OE, Kouri G, Pérez J, Martínez E, Vásquez S et al. Classical dengue hemorrhagic fever resulting from two dengue infections spaced 20 years or more apart: Havana, Dengue 3 epidemic,2001-2002. Internat J Infect Dis2005;9:281-5.

10. SetiawanM,Tatang K, Samsi TK ,Pool T,Surgianto D,Wulur H. Dengue haemorrhagic fever : ultrasound as an aid to predict the severity of disease.Pediatr Radiol.1998; 28 : 1-4.

11. Porter K, Beckett Ch, Kosasih H, Tan R, AlisjahbanaB, Rudiman P, Nidjaja $\mathrm{S}$ et al Epidemiology of dengue and dengue hemorrhagic fever in cohort of adults living in Bandung,West Java,Indonesia. AmJ Trop Med Hyg. 2005:72: 60-6

12. Smith AW, Schwartz E. Dengue in travelers. N Eng J Med, 2005;353:924-32

13. Ranjit S, Kissoon N, Jayakumar I, Aggressive management of dengue shock syndrome may decrease mortality rate: A suggested protocol. Pediatr Crit Care Med 2005;6:412-18.

14. Pizarro D. Tratamiento parenteral de la deshidratación en niños con diarrea Bol. Med Hosp Infant Mex 1986; 43: 515-22.
15. Greenough III, WB, Hirschhorn N, Gordon RS, Lindenbaun J, Ally KM. Pulmonary oedema associated with acidosis in patients with cholera. Trop Geog Med. 1976; 28:86-90

16. Roberts I .Human albumin administration in critical ill patients systematic review of randomized controlled trials B M J. 1998 ;317: 235-40

17. Simon Finfer, Rinaldo Bellomo, Neil Boyce, Julie French, John Myburgh, Robyn Norton. A comparison of albumin and saline for fluid resuscitation in intensive care unit N Engl J Med. 2004 ; 350 :2247-56

18. Rogers MC, Nichols DG. Texbook of Pediatrics Intensive Care. (3th ed) Baltimore, William \& Wilkins, 1996.

19. Nhan N, Thanh Cao Xuan Thanh Phuong, Rachel Kneen, Bridget Wills, Nguyen Van My, Nguyen Thi Que Phuong, et al. All Acute management of dengue shock síndrome a randomized double-blind comparison of 4 intravenous fluid regimens in firs hour Clin Infect Dis. 2001; 32 :204-13.

20. Harris E, Pérez L, Phares CR, Pérez MA, Idiaquez W, Rocha J, et al. Fluid intake and decreased risk for hospitalization for dengue fever, Nicaragua Emerg Infect Dis, 2003; 9:1003-06. 